\title{
Manotheranostics
}

2017; 1(4): 358-368. doi: 10.7150/ntno.21384

Review

\section{Encapsulation of Inorganic Nanomaterials inside Virus-Based Nanoparticles for Bioimaging}

\author{
Wenjing Zhang ${ }^{1,2 *}$, Chengchen $\mathrm{Xu}^{1}{ }^{1 *}$, Gen-Quan Yin ${ }^{3}$, Xian-En Zhang4, Qiangbin Wang ${ }^{5 凶}$, Feng $\operatorname{Li}^{1 凶}$ \\ 1. State Key Laboratory of Virology, Wuhan Institute of Virology, Chinese Academy of Sciences, Wuhan, 430071, China; \\ 2. University of Chinese Academy of Sciences, Beijing, 100049, China; \\ 3. Guangzhou Women and Children's Medical Center, Guangzhou Medical University, Guangzhou, 510623, China; \\ 4. National Laboratory of Biomacromolecules, CAS Center for Excellence in Biomacromolecules, Institute of Biophysics, Chinese Academy of Sciences, Beijing, \\ 100101, China; \\ 5. CAS Key Laboratory of Nano-Bio Interfaces, Division of Nanobiomedicine and i-Lab, Suzhou Institute of Nano-Tech and Nano-Bionics, Chinese Academy \\ of Sciences, Suzhou, 215123, China. \\ * These authors contributed equally to this work. \\ $\triangle$ Corresponding authors: qbwang2008@sinano.ac.cn; fli@wh.iov.cn
}

(c) Ivyspring International Publisher. This is an open access article distributed under the terms of the Creative Commons Attribution (CC BY-NC) license (https://creativecommons.org/licenses/by-nc/4.0/). See http://ivyspring.com/terms for full terms and conditions.

Received: 2017.06.08; Accepted: 2017.07.23; Published: 2017.08.18

\begin{abstract}
Virus-based nanoparticles (VNPs) can serve as containers for inorganic nanomaterials with excellent physical and chemical properties. Incorporation of nanomaterials inside the inner cavity of VNPs has opened up lots of possibilities for imaging applications in the field of biology and medicine. Encapsulation of inorganic nanoparticles (NPs) in VNPs can achieve the labeling of VNPs with nanoprobes and maintain the original outer surface features of VNPs at the same time. In return, VNPs enhance the stability and biocompatibility of the inorganic cargoes. This review briefly summarizes the current typical strategies to encapsulate inorganic nanomaterials in VNPs, i.e. mineralization and self-assembly, as well as the applications of these hybrid nanostructures in the field of bioimaging, including in vitro and in vivo fluorescence imaging, magnetic resonance imaging, and theranostics. Nanophotonic studies based on the VNP platform are also discussed. We anticipate that this field will continue to flourish, with new exciting opportunities stemming from advancements in the rational design of VNPs, the development of excellent inorganic nanomaterials, the integration of multiple functionalities, and the regulation of nano-bio interfacial interactions.
\end{abstract}

Key words: virus-based nanoparticle, encapsulation, mineralization, self-assembly, bioimaging, theranostics.

\section{Introduction}

Viruses are infectious agents in the biological sense, while in the chemical sense, they are highly ordered assemblies of biomacromolecules, with well-defined structures and monodisperse sizes in the range from tens to hundreds of nanometers. Generally, a virus has a proteinaceous capsid with the genomic nucleic acid inside, and enveloped viruses possess an additional membrane layer wrapping the capsid. Viruses are sometimes looked upon as natural nanomaterials due to their characteristic sizes and structures. Accordingly, the term virus-based nanoparticles (VNPs) have come into being. They are formulations based on viruses or virus-like particles, which are often assembled from multiple copies of viral capsid proteins (CPs) with a hollow inner cavity [1-3].

Encapsulation is a common strategy used by viruses to protect and deliver their genetic materials. In fact, it is also used by lots of other biostructures for specific biological reactions and processes under confinement, e.g. carboxysome [4], Eut microcompartment [5], and ferritin [6, 7]. In recent years, encapsulation of artificial cargoes-like inorganic nanomaterials, synthetic RNA, small molecule drugs, metal ions, and organic fluorophores in VNPs, has emerged as a unique strategy to integrate various functionalities for diverse purposes $[1,8,9]$. This review seeks to summarize the encapsulation of 
inorganic nanomaterials with emphasis on the encapsulating strategies and bioimaging applications. Encapsulation of inorganic materials in VNPs is a typical and beautiful example of the interplay between biology and nanotechnology. The inorganic cargoes render VNPs new physical or chemical properties [2, 3]. In return, VNPs increase the biocompatibility and facilitate addressable functionalization of the inorganic nanomaterials. In combination, the hybrid nanoparitcle-containing VNPs (NP@VNPs) provide a versatile nanoplatform for the fabrication of theranostic nanostructures or nanodevices.

\section{Strategies for encapsulation of inorganic nanomaterials in VNPs}

A variety of VNPs have been explored to encapsulate inorganic nanomaterials. There are two ways to load inorganic nanomaterials inside VNPs, i.e. mineralization in the inner cavity of VNPs and encapsulation of pre-formed nanomaterials through controllable self-assembly (Table 1).

\section{Mineralization}

The inner cavity of VNPs can serve as a nanoreactor for mineralization of inorganic nanomaterials with controllable sizes. In order to mineralize specifically inside VNPs, precursor metal ions should be attracted and trapped in the inner cavity rather than on the outer surface of VNPs. This can be achieved through electrostatic interaction, using ion affinity tags, or equipping seeds for mineralization.

In 1998, Douglas and co-workers first described the mineralization of paratungstate and decavanadate inside CCMV VNPs by means of electrostatic interaction. The pores on the VNP shell exhibit a $\mathrm{pH}$-dependent gating mechanism. They swelled at $\mathrm{pH}>6.5$, allowing the negatively charged polyoxometalate salts to be attracted by the positively charged interior interface, and closed at $\mathrm{pH}<6.5$, resulting in the entrapment and oligomerization of polyoxometalate salts (Fig. 1A) [10]. Similarly, $\beta-\mathrm{TiO}_{2}$ nanoparticles (NPs) were also successfully synthesized inside CCMV VNPs [11]. The charge of the interior surface of VNPs can be modified through genetic engineering to extend the spectrum of precursors. The nine basic residues at the $\mathrm{N}$-terminus of the CCMV subunit were replaced with glutamic acid to switch the interior surface of VNPs from cationic to anionic, which favored strong interaction with positively charged ferric precursors and led to size-constrained iron oxide formation inside CCMV VNPs [12]. For CPMV, the inner surface is rich in negatively charged amino acids, which has been used to mineralize iron oxide and Co NPs $[13,14]$. Electrostatic interaction has also been exploited to realize the mineralization inside the inner cavity of rod-shaped VNPs. Neutral or alkaline condition was used to reduce the positive charge of the outer surface and increase the negative charge on the inner surface of TMV VNPs. At pH 7.0, cationic silver precursors were preferentially sequestered within the central channel, which led to the growth of aligned discrete Ag NPs within the internal channel of TMV VNPs [15]. Another rod-shaped VNP, ToMV, has also been used for fabrication of $3 \mathrm{~nm}$ magnetic NPs $(\mathrm{Co} / \mathrm{Pt}$ alloy) aligned within its channel by introducing lysine facing the inner surface to increase positive charges [16]. The flexibility of VNPs to be rationally engineered to switch interior surface charges based on their structural information enriches the possibilities of synthesizing different species of NPs inside VNPs.

Genetically introducing metal affinity tags is another approach to tune inorganic NP deposition inside VNPs. P22 VNP is assembled from 420 identical copies of CP with approximately 300 copies of scaffolding protein (SP) binding on the inner surface. SP can be genetically modified with different affinity peptides to provide nucleation sites for a variety of inorganic NPs. For examples, $\mathrm{Fe}_{2} \mathrm{O}_{3}, \mathrm{TiO}_{2}$, and $\mathrm{CdS}$ NPs were successively synthesized inside P22 VNPs by this approach [17-20]. In the case of TMV VNPs, mutation of threonine 103 to cysteine dramatically enhanced the assembly capability and stability of TMV rod-like structure due to the formation of disulfide bonds between adjacent subunits [21]. Then growth of AuNP chains (AuNCs) and Au nanorods (AuNRs) was realized in the internal channel of the mutant TMVVNPs, mediated by the strong coordination interaction between $\mathrm{Au}^{3+}$ and sulfhydryl of the introduced cysteine (Fig. 1B) [22]. The single mutation of cysteine 103 successively played two important roles, namely, stabilizing TMV rod-like template and mediating the deposition of Au.

Equipping inorganic seeds on the inner surface is an alternative route for NP mineralization inside VNPs. Taking the advantages of natural affinity to $\mathrm{Pd}$ or $\mathrm{Pt}$, a series of nanowires (NWs), such as $\mathrm{Ni}, \mathrm{Co}, \mathrm{Cu}$, $\mathrm{Co} / \mathrm{Fe}, \mathrm{Co} / \mathrm{Pt}$, and $\mathrm{FePt}_{3} \mathrm{NWs}$ of $3 \mathrm{~nm}$ in diameter and a few hundred nanometers in length have been synthesized inside the central channel of TMV VNPs after the activation of $\mathrm{Pd}$ or $\mathrm{Pt}$ followed by electroless deposition [23-27]. In addition to direct nucleation of seeds on the inner surface, preformed NPs can also be equipped inside the inner cavity of VNPs as seeds for subsequent growth of NPs. Most recently, we have established such a route with SV40 VNPs to mineralize AuNPs and Au@Ag core-shell NPs. A preformed $5 \mathrm{~nm}$ AuNP was encapsulated inside SV40 
VNP as the seed, which was followed by further growth of an outer metal layer. This method circumvents the limitation that only gentle reaction conditions can be used for seed synthesis inside viral protein cages to avoid protein impairment. The encapsulation of the preformed $5 \mathrm{~nm}$ seed was realized by virtue of the self-assembly process of SV 40
VNP, which will be discussed in the next section [28].

It is worth mentioning that in addition to VNPs, other protein cages, for example, the ferritin family, have also been versatile templates for constrained synthesis of nanomaterials. There are several excellent reviews to be referred to in this regard $[29,30]$.

Table 1. A brief summary of NP@VNPs and their bioimaging applications.

\begin{tabular}{|c|c|c|c|}
\hline Viruses & $\begin{array}{l}\text { Encapsulation } \\
\text { strategies }\end{array}$ & Encapsulated inorganic nanomaterials & Applications in bioimaging \\
\hline \multirow[t]{2}{*}{ CCMV(cowpea chlorotic mottle virus) } & Mineralization & $\begin{array}{l}\text { Paratungstate and decavanadate [10], } \beta-\mathrm{TiO}_{2}[11] \text {, iron oxide } \\
\text { [12] NPs }\end{array}$ & \\
\hline & Self-assembly & AuNP [63-65] & \\
\hline BMV(brome mosaic virus) & Self-assembly & $\begin{array}{l}\text { AuNP }[31,33,42,46,60,66], \text { iron oxide NP }[43,56], \text { CdSe@ZnS QD } \\
{[32]}\end{array}$ & Nanophotonics [60], MRI $[43,56]$ \\
\hline CPMV(cowpea mosaic virus) & Mineralization & CoNP $[14]$ & \\
\hline $\begin{array}{l}\text { RCNMV(red clover necrotic mosaic } \\
\text { virus) }\end{array}$ & Self-assembly & $\mathrm{Au}, \mathrm{CoFe}_{2} \mathrm{O}_{4}$, and CdSe NPs $[37,38]$ & \\
\hline MS2(bacteriophage) & Self-assembly & AuNP [62], CdSe@ZnS QD [67] & Nanophotonics [62] \\
\hline P22 (bacteriophage) & Mineralization & $\mathrm{Fe}_{2} \mathrm{O}_{3}[17], \mathrm{TiO}_{2}[18], \mathrm{CdS}[19,20] \mathrm{NPs}$ & \\
\hline \multirow[t]{2}{*}{ SV40 (simian virus 40 ) } & Mineralization & AuNP and Au@AgNP [28] & \multirow{2}{*}{$\begin{array}{l}\text { Fluorescence imaging in cells [49] and } \\
\text { in vivo [50], MRI [68], nanophotonics } \\
{[61] \text {, theranostics [59] }}\end{array}$} \\
\hline & Self-assembly & $\begin{array}{l}\text { CdSe@ZnS QD [35,49,59,61,69,70], AuNP [69,71], } \mathrm{Ag}_{2} \mathrm{~S} \text { QD } \\
{[50,69], \mathrm{MNP}[68]}\end{array}$ & \\
\hline $\begin{array}{l}\text { HBV (hepatitis B core virus-like } \\
\text { particles) }\end{array}$ & Self-assembly & $\mathrm{Fe}_{3} \mathrm{O}_{4} \mathrm{NP}[41], \mathrm{FeO} / \mathrm{Fe}_{3} \mathrm{O}_{4} \mathrm{NP}[72], \mathrm{\gamma}^{-} \mathrm{Fe}_{3} \mathrm{O}_{4} @ \mathrm{AuNP}$ [57] & MRI $[41,57]$ \\
\hline Alpha-virus (nucleo-capsid) & Self-assembly & AuNP [73] & \\
\hline $\begin{array}{l}\text { PTLV (pseudotyped HIV-1-based } \\
\text { lentivirus) }\end{array}$ & Self-assembly & CdSe@ZnS QD [39] & Fluorescence imaging in cells [39] \\
\hline $\begin{array}{l}\text { HIV-1 (human immunodeficiency } \\
\text { virus type } 1 \text { ) }\end{array}$ & Self-assembly & CdSe@ZnS QD [40] & Fluorescence imaging in cells [40] \\
\hline TMV (tobacco mosaic virus) & Mineralization & $\begin{array}{l}\mathrm{Ni}[24,25], \mathrm{Co}[24], \mathrm{Cu}[27], \mathrm{Co} / \mathrm{Fe}[26], \mathrm{Co} / \mathrm{Pt} \text { and } \mathrm{FePt}_{3} \mathrm{NWs} \\
{[23], \text { aligned AgNPs [15], } \mathrm{Au} \mathrm{NRs}[22]}\end{array}$ & \\
\hline ToMV (tomato mosaic virus) & Mineralization & $\mathrm{Co} / \mathrm{Pt} \mathrm{NP}[16]$ & \\
\hline
\end{tabular}

A

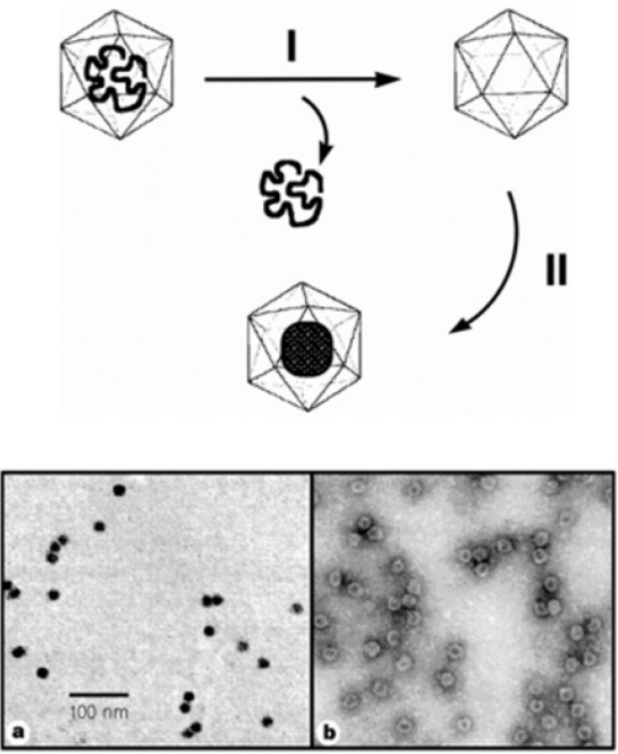

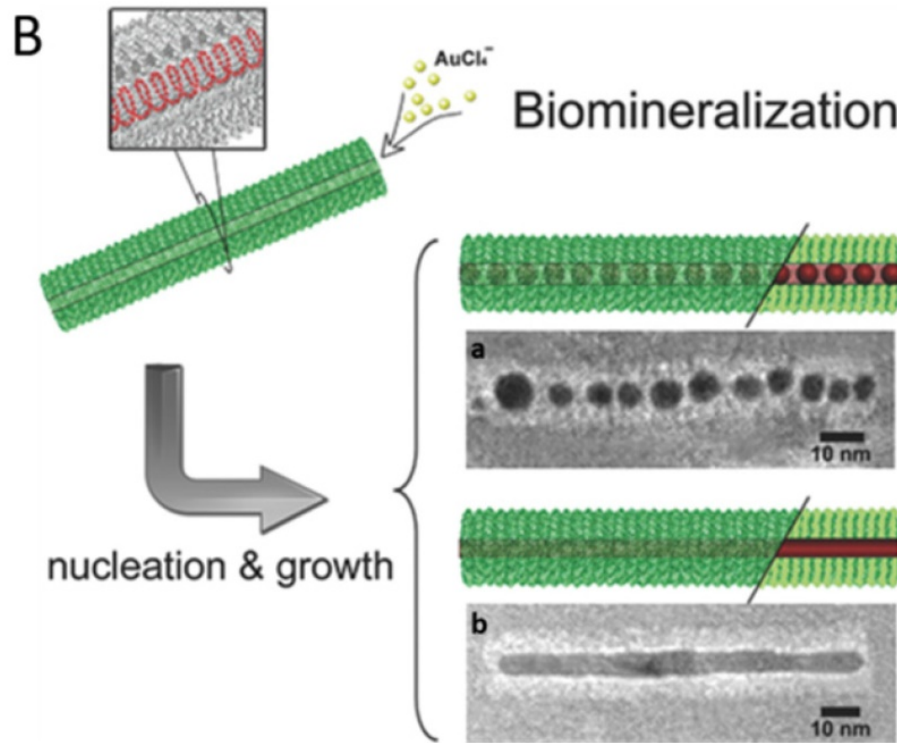

Figure 1. Encapsulation of nanomaterials inside VNPs by mineralization. A) Upper: Schematic illustration of the synthetic approach for mineralization within the CCMV VNPs. Step I involves the removal of viral RNA and purification of the empty VNPs by sucrose gradient centrifugation. Step II involves the selective mineralization of an inorganic paratungstate species within the confines of the VNPs, at $\mathrm{pH} 6.5$ and $6^{\circ} \mathrm{C}$. Lower: TEM images of paratungstate-mineralized VNPs. a) An unstained sample showing discrete electron dense cores; b) A negatively stained sample of (a) showing the mineral core surrounded by the intact VNP protein cage. Scale bar: $100 \mathrm{~nm}$. Reproduced with permission [10]. B) Site-specific biomineralization of gold nanostructures in TMV rod-like VNPs. The cysteines arrayed in the interior of mutated nanotubes are specifically bound with the gold precursors, which initializes the controlled growth of AuNCs (a) or AuNRs (b). Scale bars: 10 nm. Reproduced with permission [22]. 


\section{Self-assembly}

VNPs can undergo the disassembly-assembly cycle controlled by buffer conditions, which has been widely utilized to encapsulate guest cargoes. Inorganic nanomaterials of different surface properties, components, and sizes have been encapsulated into VNPs of different viruses (Table 1).

For encapsulation in VNPs, the surface coatings of inorganic NPs, which play an important role in determining the interactions between $\mathrm{CPs}$ and inorganic NPs, have been the primary consideration. What surface properties are required for an inorganic NP to be encapsulated by VNPs? The central idea is to mimic the packaging of viral genome. Therefore, negatively charged coatings are often used to make NPs to mimic viral nucleic acids to initiate the assembly of VNPs. However, different species of negatively charged surface coatings may present different performance in initiating the assembly of VNPs. In an early report by Chen et al, AuNPs coated by carboxylate-terminated thiolalkylated tetraethylene glycol (TEG) reached a high encapsulation efficiency of $95 \%$ by BMV VNPs. However, in the case of citrate-coated AuNPs, serious aggregation occurred during the reassembly procedure (Fig. 2A) [31]. Similarly, quantum dots (QDs) coated by HS-poly(ethylene glycol) (PEG)-COOH showed the highest ability in promoting BMV VNP assembly in comparison to QDs functionalized with phospholipid micelle, streptavidin-biotin-DNA, or dihydrolipoicacid (DHLA) [32]. Those surface coatings, which can keep the stability of inorganic NPs as well as provide efficient nucleation and/or propagation sites for the assembly of VNPs, are excellent in promoting the encapsulation process [31]. Furthermore, the effect of surface charge density of NPs on the encapsulation by BMV VNPs was studied with AuNPs functionalized by a mixture of HS-TEG-COOH and HS-TEG-OH. It was found that a critical surface charge density was required for BMV VNP assembly [33]. According to these experimental observations, the authors presented a model to describe the encapsulation of spherical particles functionalized with weakly acidic surface groups, which demonstrated that surface charge regulation in combination with size polydispersity of the NPs has a large effect on the encapsulation efficiency [34]. In contrast, a quite different case is presented by SV40 VNPs. It showed that the encapsulation of NPs does not rely on the surface charge of NPs, as negatively charged mercaptopropanoic acid (MPA)-coated QDs and DNA-coated QDs, neutrally charged methoxy-terminated PEG-coated QDs, and positively charged amine-terminated PEG-coated QDs could be encapsulated by SV40 VNP with comparable efficiencies [35]. The lack of dependence of NP encapsulation by SV40 VNP on electrostatic interaction may be related to the unique structural organization of natural SV40 virion. SV40 genome DNA forms a minichromosome with host histones that neutralize the negative charges of DNA, and the interaction between the capsid and the minichromosome is mediated by two minor CPs [36]. Such a structure feature suggests that genome DNA encapsulation of SV40 depends much less on electrostatic attraction than those of viruses like CCMV and BMV.

Packaging signals of specific nucleotide sequences have evolved in many viruses to guide selective encapsulation of viral genomes. Such specific interactions between nucleic acids and CPs have been exploited to direct NP encapsulation by VNPs. As a proof of concept, oligonucleotide mimics of the origin of assembly sequence (OAS) in RCNMV were attached to NPs to recruit RCNMV CPs and to direct the assembly of VNPs. Using this strategy, $\mathrm{Au}$, $\mathrm{CoFe}_{2} \mathrm{O}_{4}$, and CdSe NPs ranging from $3 \mathrm{~nm}$ to $15 \mathrm{~nm}$ in diameter were successfully encapsulated inside RCNMV VNPs (Fig. 2B). Attempt to encapsulate NPs larger than $17 \mathrm{~nm}$ did not result in well-formed RCNMV VNPs, which is consistent with the presence of a $17 \mathrm{~nm}$ cavity in native viron $[37,38]$. This strategy has also been used to encapsulate NPs inside enveloped virus in living cells. QDs conjugated with modified genomic RNAs (gRNAs), were packaged into enveloped virus together with the gRNAs (Fig. 2C) [39]. Besides oligonucleotide sequences, other inner components of virus can also be used to mediate the encapsulation of NPs. Recently, QDs modified with the accessory protein $\mathrm{Vpr}$ have been encapsulated into HIV-1 virons during virus assembly [40]. In addition to learning from natural packaging mechanisms, other specific interactions have also been utilized to facilitate the encapsulation of NPs by VNPs. For example, nickel-nitrilotriacetic acid (Ni-NTA) functionalized $\mathrm{Fe}_{3} \mathrm{O}_{4} \quad$ NPs efficiently templated the assembly of HBVVNPs with carboxyl-terminally appended polyhistidine tags [41].

The size of inorganic NPs is another factor affecting the encapsulation by VNPs. AuNPs with different diameters guided the assembly of BMV VNPs into $T=1, T=2$ or $T=3$ icosahedral structures, respectively, resembling to the three classes of BMV viral particles found in host cells (Fig. 2D) [42]. Phospholipid micelle-coated magnetic NPs which was larger than the inner cavity of native BMV capsid was also found to be able to template the assembly of BMV CPs into VNPs larger than the native $T=3 \mathrm{BMV}$ 
viron [43]. For HBV VNPs, the VNP diameter followed the increase of cargo size linearly until it reached the value expected for the $T=4$ capsid, and then remained unchanged upon further increase of the cargo diameter up to $18 \%$ [44]. The flexibility of
VNPs to encapsulate various sizes of NPs to form polymorphic assemblies, makes them more versatile in biomedical applications, because shape and size are two important factors that affect the in vivo behaviors of VNPs [45].
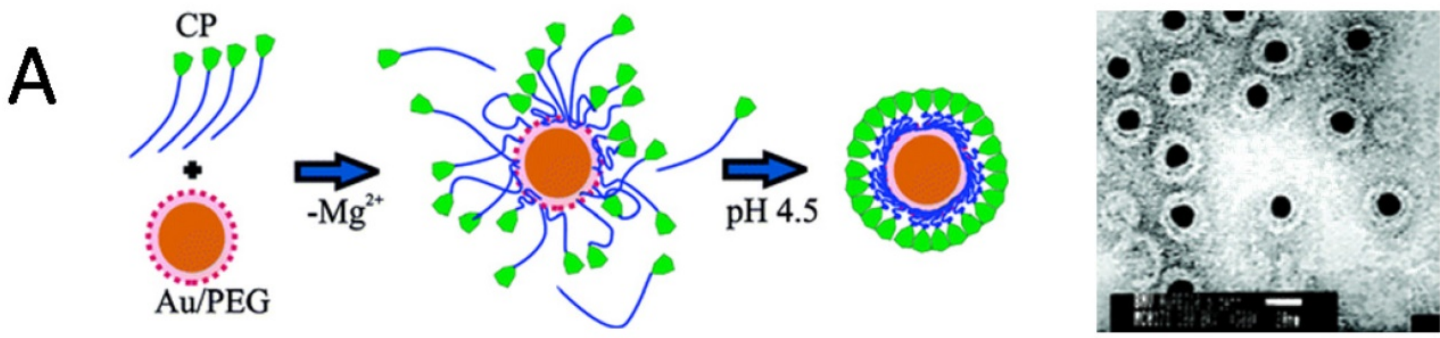

\section{B}
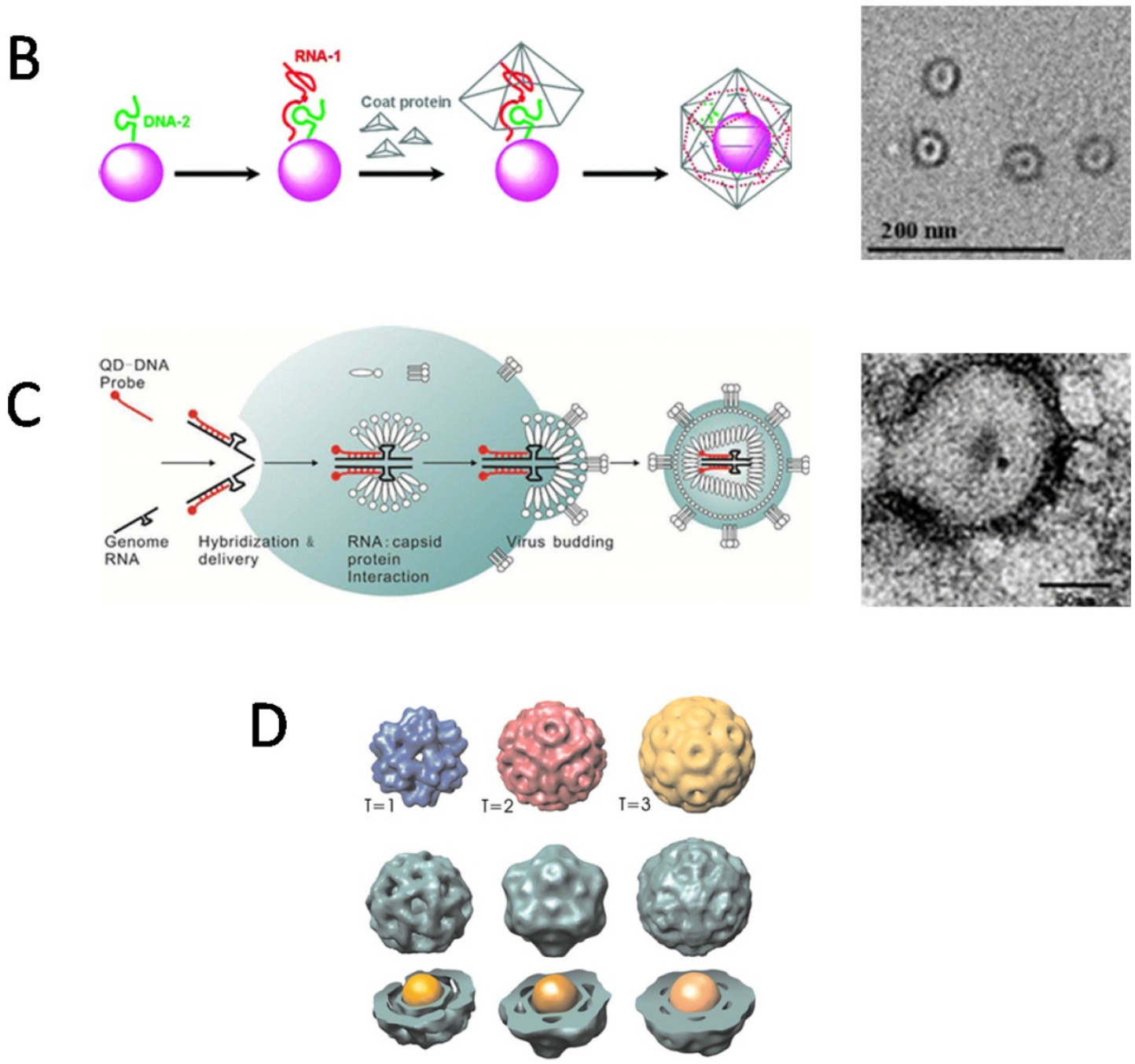

Figure 2. Encapsulation of nanomaterials inside VNPs by self-assembly. A) Electrostatic interaction mediated the encapsulation of AuNPs in BMV VNPs. Left: A proposed mechanism shows electrostatic interaction-mediated formation of disordered CP-AuNP complexes followed by a crystallization phase in which the CP-CP interactions lead to the formation of a regular VNP. Right: Negative-staining TEM image of BMV VNPs with a $12 \mathrm{~nm}$ AuNP core. Scale bar, $20 \mathrm{~nm}$. Reproduced with permission [31]. B) Artificial OAS guided the encapsulation of inorganic NPs by RCNMV VNPs. Left: The encapsulation procedure includes functionalization of NPs with DNA-2 and RNA-1 to form OAS, recruitment of CPs by OAS, and formation of VNPs with a NP inside. Right: Negative-staining TEM image of 4 nm CoFe $\mathrm{O}_{4}$ NPs encapsulated inside RCNMV VNPs. Scale bar, $200 \mathrm{~nm}$. Reproduced with permission [38]. C) QDs were encapsulated intracellularly by the capsid of VSV-G PTLV. Left: Schematic of the encapsulation process. QDs carrying packaging signal oligonucleotides are co-transfected with the packaging plasmids of VSV-G PTLV into host cells. Then the QDs are encapsulated into the viral capsid together with the genome RNAs during virus budding. Right: Negative-staining TEM image of VSV-G PTLV with QDs encapsulated inside. Scale bar: $50 \mathrm{~nm}$. Reproduced with permission [39]. D) Size of the AuNP core controlled the assembly of VNPs. Encapsulation of AuNPs of $6 \mathrm{~nm}, 9 \mathrm{~nm}, 12 \mathrm{~nm}$ led to VNPs resembling $T=1$, pseudo $T=2$, and $T=3$ capsids of BMV, respectively. Reproduced with permission [42]. 
Besides the surface properties and size effect of cargo NPs, environmental parameters also play a role in the encapsulation by VNPs. For instance, the buffer conditions, which not only control the disassembly-assembly cycle, but also influence the assembly pathway. It was reported that depending on the $\mathrm{pH}$ value and ionic strength, the assembly of BMV VNPs around anionic AuNPs could be cooperative or non-cooperative adsorption. At high $\mathrm{pH}$ and low ionic strength, non-cooperative Langmuir adsorption of the BMV CPs around the anionic core occurred, whereas at low $\mathrm{pH}$ and low ionic strength, initial cooperative $\mathrm{CP}$-AuNP association followed by $\mathrm{CP}$ rearrangement took place to form a regular capsid-like structure [46]. In addition, the buffer conditions may influence encapsulation by affecting cargo stability. Some protein cages, such as MS2 VNP and ferritin, need an ultra-low $\mathrm{pH}$ treatment to disassemble. When NPs are mixed with the disassembled CP oligomers for encapsulation, they would be prone to precipitation and fail to be encapsulated. This problem can be solved by finding out either proper surface coatings of NPs or new strategies to control the disassembly-assembly cycle of VNPs.

\section{NP@VNPs hybrid nanostructures for bioimaging}

Encapsulation of inorganic nanomaterials in VNPs has inspired people to come up with new ideas about biolabeling and bioimaging. In this section, we review the recent advances of bioimaging applications of NP@VNPs, including live cell imaging, in vivo imaging, magnetic resonance imaging (MRI), and nanophotonics. The most recently designed hybrid multifunctional NP@VNPs as a novel nanotheranostic tool is introduced. Future opportunities and challenges of NP@VNPs in bioimaging field are also discussed.

\section{Fluorescence imaging}

Fluorescence imaging is now an evolving field that has already achieved significant advances, due to the accumulative availability of fluorescent proteins, dyes and probes, as well as the development of optical imaging technologies [47]. As a typical representative fluorescent nanoprobes, QDs emit in the visible and near infrared wavelength region with high brightness and excellent photostability [48]. However, it has been controversial that labeling QDs on virus or VNP outer surfaces would affect virus-host cell interactions and might cause artifacts. Therefore, encapsulation of QDs in VNP cavities provides an alternative way that has attracted increasing interests.
The first demonstration of imaging viral behaviors in living cells with encapsulated QDs was reported with the self-assembling VNPs of SV40 in 2009. By real-time and long-term tracking of the cellular uptake and intracellular transportation, it was found that SV40 QD@VNPs was internalized by cells through caveolae-mediated endocytosis, then travelled along the microtubules and accumulated in the endoplasmic reticulum, like the wild-type SV40 (Fig. 3A) [49], suggesting that the early infection steps of SV40 are basically determined by the major CP VP1.

Encapsulation of QDs inside VNPs eliminates in principle the disadvantages caused by QD modification on outer surface of viruses and avoids controversies over the modification strategies. QD encapsulation therefore enables high-fidelity imaging, which should benefit the investigation of infection events of other viruses. However, the most challenging is to label and track live viruses, not merely VNPs, using such a strategy.

Subsequent efforts succeeded in encapsulating QDs inside the core of vesicular stomatitis virus glycoprotein (VSV-G) pseudo-typed HIV-1-based lentivirus (PTLV) during the assembly process of the viruses in living cells. Revealed by the real-time single particle tracking of QD@PTLVs, there was significant colocalization between the virus and early endosomes. In the following steps, the trajectory and velocity of single virus particle on microtubules were recorded, and a microtubule-dependent QD@PTLVs cellular entry route was clearly drawn [39]. Most recently, productive entry of HIV-1 into human primary macrophages has been fluorescently tracked at the single particle level by taking advantages of encapsulated QDs, which revealed that the infection process requires clathrin-mediated endocytosis, endosomal escape of the viral core, and actin dynamics (Fig. 3B) [40]. Both studies are successful examples of live virus labeling and imaging by the QD encapsulation strategy. The high brightness and photostability of QDs endow real-time virus tracking to the single particle level.

When in vivo deep tissue imaging is needed, challenges of high absorbance, serious scattering and strong autofluorescence of the tissue to the visible fluorescence should be overcome to reach deep tissue imaging with desired temporal and spatial resolution. The second near infrared (NIR-II) optical window $(1000-1700 \mathrm{~nm})$ of tissues has been utilized as a solution to the problem, because tissues have minimal optical extinction and autofluorescence in the NIR-II range. $\mathrm{Ag}_{2} \mathrm{~S}$ QDs have proved to be an excellent NIR-II probe with high brightness, stability, and good biocompatibility. By encapsulating the $\mathrm{Ag}_{2} \mathrm{~S}$ QDs, 
dynamics and biodistribution of SV40 VNPs in live mice were clearly monitored in real time. It was found that $\mathrm{Ag}_{2} \mathrm{~S} @ V N P$ s quickly accumulated in liver, spleen, and bone marrow within 5 min post injection. Surface PEGylation of VNPs showed comprehensive impact on the in vivo behaviors of SV40 VNPs, including greatly elongated blood circulation time and significantly reduced accumulation in the reticuloendothelial system (Fig. 3C). Encapsulation of $\mathrm{Ag}_{2} \mathrm{~S}$ QDs enabled real-time monitoring of surface chemistry-dependent in vivo behaviors of VNPs with unprecedented spatiotemporal resolution [50]. The hybrid $\mathrm{Ag}_{2} \mathrm{~S} @$ VNPs might be a useful nanoplatform for future theranostics.

\section{Iron oxide NP@VNPs for magnetic resonance imaging}

MRI is now an advanced technique very useful in biomedical research and disease diagnosis. It is a non-invasive imaging tool that produces three dimensional anatomical images without damaging radiation. Most MRI contrast agents enhance imaging contrast by changing the relaxation rates $\left(1 / T_{1}\right.$ or $1 / T_{2}$ ) of tissue water protons, therefore improving the sensitivity and specificity for detection [51]. Viral capsids provide the advantages of suitable sizes and shapes that can increase the chances to enhance the relaxation rates as well as an ideal modification platform for contrast agents labeling [52]. In 2005, Douglas et al. developed VNPs as MRI contrast agents by chemically attaching $\mathrm{Gd}^{3+}$ to CCMV VNPs [53]. Subsequently, several other VNPs have been investigated as potential MRI contrast agents labeled with $\mathrm{Gd}^{3+}$. Detailed labeling strategies and applications of $\mathrm{Gd}^{3+}$-VNPs contrast agents have been summarized in recent review papers [3,9,54,55]. Here we concentrate on diverse MRI applications of encapsulated iron oxide NPs in VNPs.
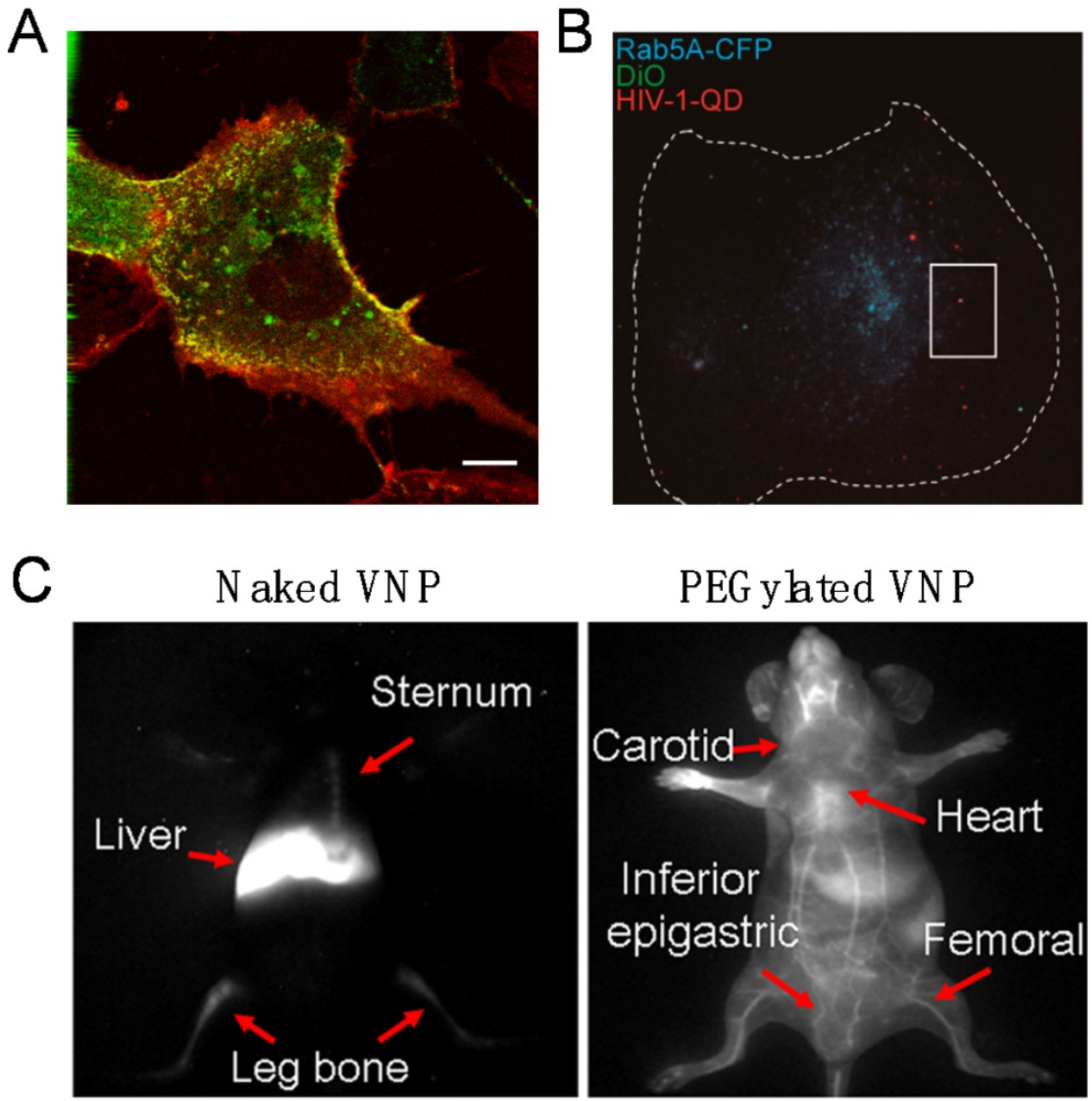

Figure 3. Real-time fluorescence bioimaging through encapsulation of QDs in VNPs. A) Live fluorescence microscopy of Vero cells transiently expressing caveolin-1-CFP and incubated with SV40 QD@VNPs, showing that SV40 QD@VNPs (red) colocalized with the caveolae marker, caveolin-1 (green). Scale bar: $10 \mu \mathrm{m}$. Reproduced with permission [49]. B) Visualization of HIV-1 dynamic productive entry via fusion of the viral envelope with Rab5A-positive endosome. The cellular boundary of the macrophage is highlighted by a dashed line. Dual-labeled QD@HIV-DiO (green and red) was shown to colocalize with ECFP-Rab5A-marked endosome (blue) in a macrophage. Scale bar: $10 \mu \mathrm{m}$. Reproduced with permission [40]. C) In virtue of encapsulation of the Ag2S QDs, NIR-II fluorescence imaging clearly distinguished the real-time distribution of the naked and PEGylated VNPs of SV40 in living mice at $12 \mathrm{~h}$ post injection. Reproduced with permission [50]. 
While $\mathrm{Gd}^{3+}$ is the most popular agent for positive MRI contrast, superparamagnetic iron oxide can effectively reduce the transverse relaxation time $\left(T_{2}\right)$, producing negative contrast effect [54]. Application of VNPs-encapsulated iron oxide for MRI was firstly reported in 2007. Iron oxide NPs of tunable sizes were packaged inside icosahedral BMV VNPs via in vitro self-assembly [43]. The behaviors of iron oxide-encapsulating BMV VNPs in the host plant were examined. In this work, the cubic iron oxide exhibited outstanding characteristics as a negative contrast agent in two aspects. Firstly, the $18.6 \mathrm{~nm}$ cubic iron oxide NPs with enhanced charge density was chosen as inner core that could increase the stability of BMV VNPs and encapsulation efficiency. Secondly and more importantly, the cubic iron oxide NPs have much higher $r_{2} / r_{1}$ (transverse/longitudinal relaxivity) ratio than that of commercial contrast agents with similar sizes such as Feridex ${ }^{\circledR}$ and Supravist ${ }^{\circledR}$, and other $\mathrm{Gd}^{3+}$-labeled VNPs. By taking transport assay inside $N$. benthamiana leaves, it was demonstrated that iron oxide-encapsulating VNPs penetrated cell wall into cytoplasm and cell junctions of intercellular space, and thus took cell-to-cell long-range transport, while free iron oxide NPs were blocked at the exterior of plant cells (Fig. 4A) [56]. This VNP-based contrast agent may be meaningful for investigation of plant growth and development. Also, it offers a new opportunity for high-performance contrast probes design based on VNP platforms. Certainly, the size of the iron oxide core encapsulated in the VNPs should be considered to maintain natural structure of viral capsids [43]. For example, a well-designed core-shell NP, $\gamma-\mathrm{Fe}_{3} \mathrm{O}_{4} @ \mathrm{AuNP} 11 \mathrm{~nm}$ in size was optimal for VNP encapsulation and presented significantly higher $r_{2} / r_{1}$ ratio at 115.8 [57].

Due to their high MRI performance, iron oxide NP@VNPs have proved to be promising MRI contrast probes for tumor diagnosis. For instance, the transverse relaxivity $\left(r_{2}\right)$ values of above mentioned $\mathrm{Fe}_{3} \mathrm{O}_{4} @ \mathrm{HBV}$ VNPs were measured to be $98 \mathrm{mM}^{-1} \mathrm{~s}^{-1}$, $107 \mathrm{mM}^{-1} \mathrm{~s}^{-1}$, and $158 \mathrm{mM}^{-1} \mathrm{~s}^{-1}$ for $3.4 \mathrm{~nm}, 6.1 \mathrm{~nm}$, and $11.7 \mathrm{~nm} \mathrm{Fe}_{3} \mathrm{O}_{4}$ cores, respectively. They were verified as potential MRI contrast agents for tumor diagnosis using cellular uptake assays against HeLa cells (Fig. 4B) [41]. Although the potentials of iron oxide NP@VNPs in MRI and multifunctional platforms have been recognized, studies are currently still at the cellular level. In vivo investigations are needed to fulfill the potentials, along with the development of new high-performance MRI contrast NPs.

\section{NP@VNPsas theranostic platforms}

The advantages of VNPs provide possibilities to integrate different types of imaging modalities in one complex. The establishment of hybrid VNPs platforms not only offers a way to circumvent the limitations of individual imaging methods, but also promotes the detection efficiency and accuracy. Currently, designed hybrid VNPs platforms have remarkably accelerated their applications in multiple disciplines $[3,8,55,58]$.

Most recently, the hybrid QD@VNPs from SV40 discussed above was further designed for targeting, imaging, and treatment of atherosclerosis in vivo. The QD@VNPs was installed with three different functional ligands to recognize three sequential periods of atherosclerosis in original, developmental, and late stage, respectively (Fig. 5). These targeting structures effectively differentiated each period of atherosclerosis by fluorescence imaging in living mouse model, which was confirmed by ex vivo fluorescence imaging of cryo-sectioned aortas. Moreover, an antithrombin peptide, Hirulog, was attached to the VNPs surface. The antithrombin activities in target aortas of atherosclerotic were also
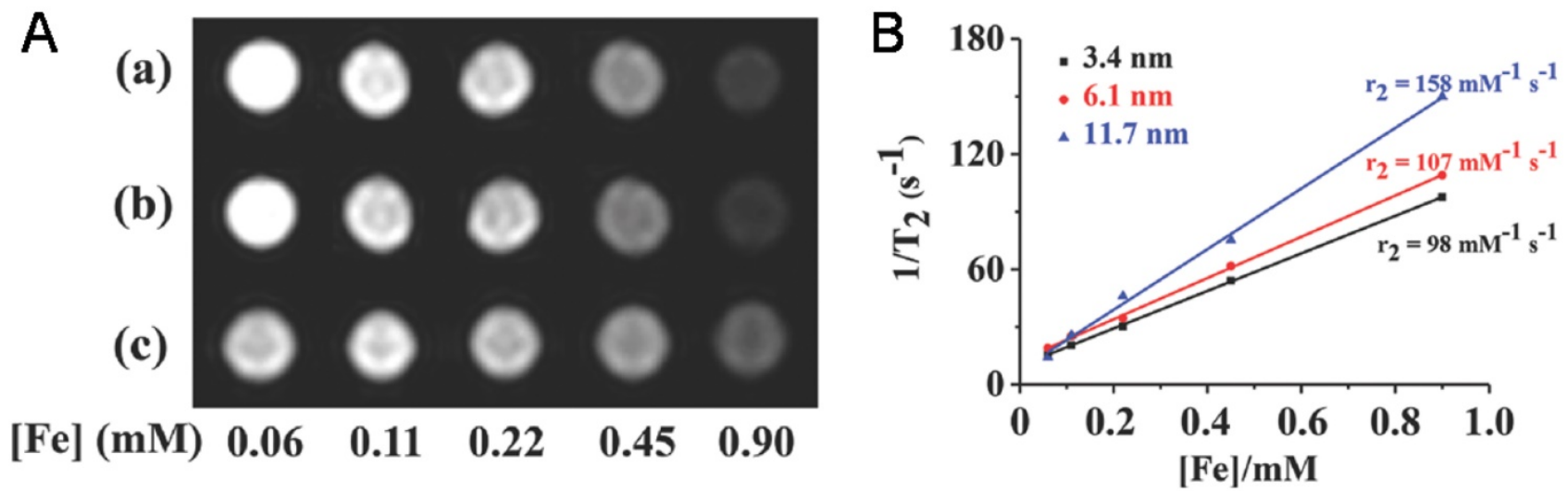

Figure 4. Iron oxide NPs encapsulated in VNPs as MRI contrast reagents. A) $T_{2}$-weighted MR images of HBV VNPs encapsulating a) 3.4nm, b) 6.1nm, and c) $11.7 \mathrm{~nm}$ of $\mathrm{Fe}_{3} \mathrm{O}_{4} \mathrm{NPs}$. Increasing the Fe concentration significantly changes the signal intensity in the MR images. $\mathrm{B}$ ) $\mathrm{Plot}$ of $1 / T_{2}$ versus Fe concentration of the Fe $\mathrm{O}_{4} \mathrm{NPs}$ of various sizes encapsulated in HBV VNPs with the slope indicating the specific relaxivity $(r 2)$. Reproduced with permission [41]. 
verified [59]. This work integrates three functionalities including fluorescent imaging, molecular targeting, and drug delivery, and represents an important step in developing multifunctional NP@VNPs for theranostics. More endeavors are further needed to advance such kinds of designer nanostructures to clinical practices.

\section{NP@VNPs for nanophotonics}

As VNPs are highly symmetrical and monodisperse in a suitable size range, they provide an ideal design platform to explore the photonic interactions between metal NPs and/or fluorophores.

The first observation on the coupling of surface plasmon resonance (SPR) in AuNP@VNPs was taken as an obvious color change caused by encapsulation of multiple AuNPs in a single VNP [60]. Later, interactions between AuNPs and QDs were described from a quantitative view based on a hybrid AuNP-QD system templated by SV40 VNPs, in which AuNPs were assembled onto the outer surface of SV40 VNPs encapsulating a QD in the cage. By tuning the number of AuNPs on QD@VNPs from one to twelve, the optical interaction between QDs and AuNPs could be quantitatively evaluated. It was observed that the fluorescence of QDs decreased dramatically with increasing AuNP:QD ratio. No fluorescence enhancement was observed because of the long distance of $8 \mathrm{~nm}$ between AuNPs and QDs (Fig. 6A) [61]. Later, Francis et al. designed a well-defined hybrid system for analyzing the interactions caused by nanoscale distance between NPs and fluorescent organic dyes. They firstly encapsulated AuNPs within the VNPs of bacteriophage MS2, and then introduced aniline groups by inserting $p$-aminophenylalanice amino acid to connect exterior dye-labeled DNA strands. By adjusting the length of DNA to control the distance between dyes and AuNPs, AuNP-enhanced fluorescence was observed using a total internal reflection fluorescence (TIRF) microscope (Fig. 6B) [62]. Herein, by encapsulating the AuNPs, MS2 VNPs served not only as a scaffold for controllable docking of fluorescent dyes, but also as a physical layer to avoid direct sticking of the dyes onto AuNPs, leading to fluorescence quenching.

\section{Conclusions and Outlooks}

It has been nearly twenty years since the first study on loading inorganic nanomaterials in VNPs was reported. Great potentials have been recognized with these protein-inorganic hybrid nanostructures. This review has briefly summarized the development of inorganic NP encapsulation inside VNPs and bioimaging applications thereof. Various methods have been established for NP encapsulation, which can be categorized into mineralization and self-assembly. The fabricated hybrid NP@VNP nanostructures have shown unique advantages in bioimaging applications in terms of sensitivity, resolution and fidelity.

Although NP@VNPs provide a promising platform for theranostic purposes, their biomedical application is still in its infant stage. Many of the bioimaging studies reported so far have been conducted in vitro, with only a few performed in living animal models. Currently, a major challenge is to target NP@VNPs to a specific region of a living body. Investigations in interactions between VNPs and biomolecules in body fluid, in vivo stability and fates of NP@VNPs, as well as the rational design of VNPs will help to find out solutions. At the same time, more endeavors are needed to integrate multiple functionalities into NP@VNPs to meet clinical requirements. Owing to the addressability, versatility, and designability of the VNP nanoplatform, these challenges will be hopefully overcome.
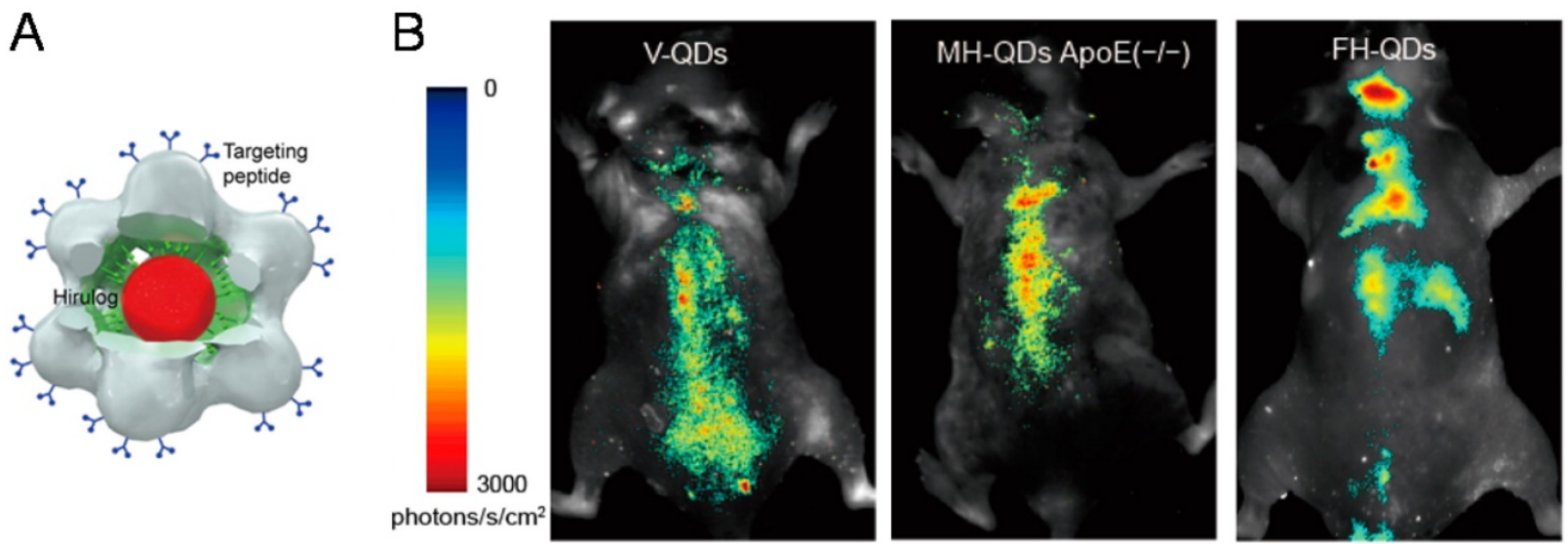

Figure 5. Trifunctional QD@VNPs of SV40 for atherosclerosis theranostics. A) Model of the trifunctional VNP, which encapsulates a QD (red) inside, is equipped with targeting peptide (blue) on the outer surface, and carries Hirulog peptide (green). B) In vivo fluorescence imaging of atherosclerosis in live ApoE(-/-) mice, using VNPs decorated with different targeting peptide for differentiating origination, development, and late stages of atherosclerosis. Reproduced with permission [59]. 
A

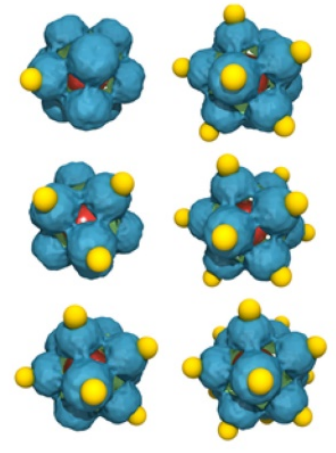

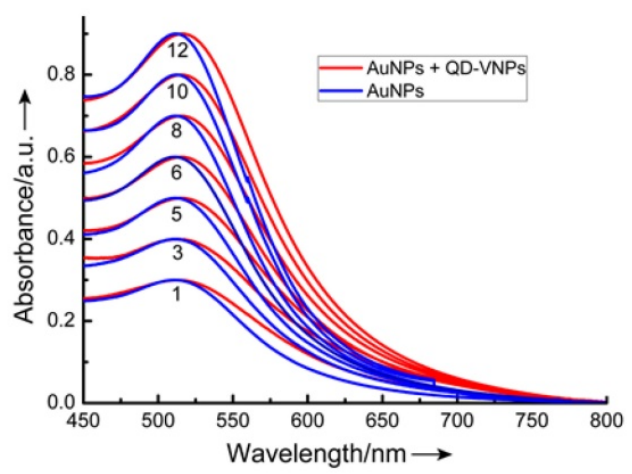

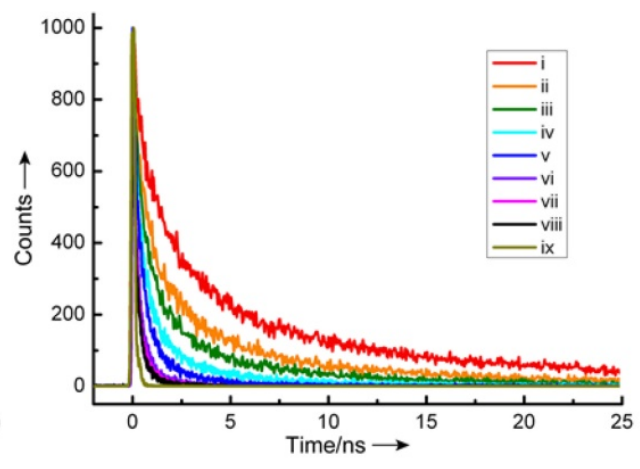

B

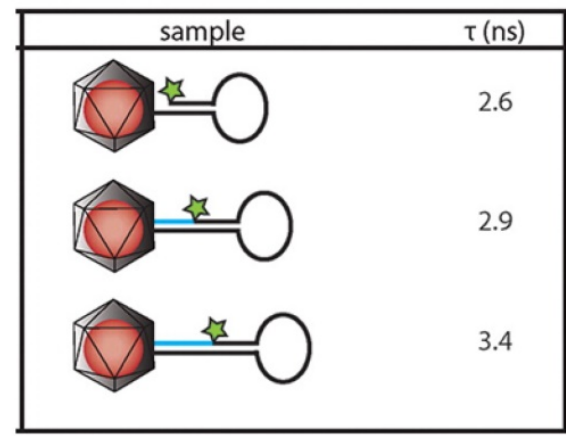

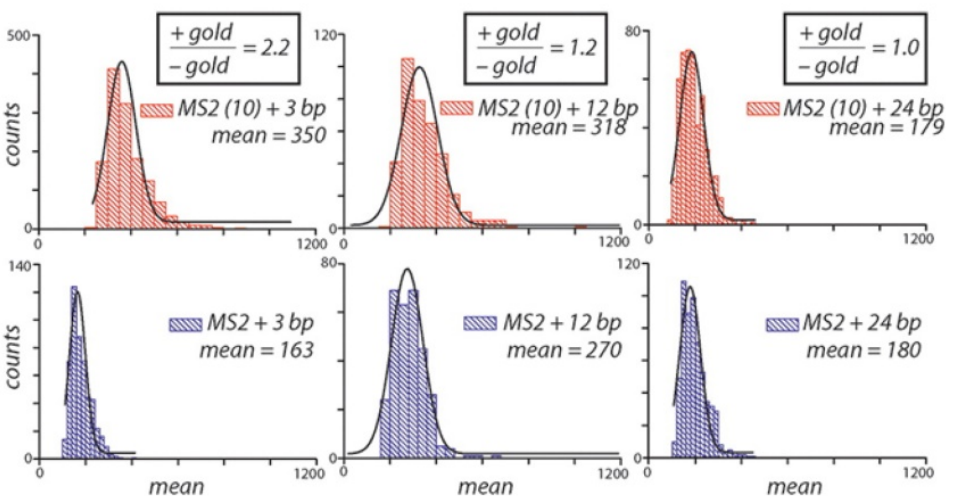

Figure 6. NP-NP and NP-dye photonic interactions investigated using the NP@VNPs platform. A) In SV40 VNP-templated 3D discrete hybrid AuNP (yellow)/QD (red) nanoarchitectures (Left), very weak SPR coupling of AuNP clusters (Middle) and AuNP number-dependent shortening of life-time of QD fluorescence (Right) were observed. Curves i-viii correspond to the number of AuNPs per VNP being 1, 3, 5, 6, 8, 10 and 12, respectively; ix, instrument response. Reproduced with permission [61]. B) Dye-labeled DNA was attached to the exterior of MS2 VNPs that encapsulate a $10 \mathrm{~nm}$ AuNP. The dye-AuNP distance was tuned using DNA strands with different lengths. The fluorescence life-time (T) of the dye was shortened as the dye and AuNP got closer (Left). Distance-dependent fluorescence enhancement of the dye by AuNPs was quantitatively determined using TIRF microscopy (Right). Enhancements of 2.2-fold and 1.2-fold were observed for 3 bp and 12 bp separations, respectively, while a 24 bp separation showed no effect. Reproduced with permission [62].

\section{Acknowledgements}

We greatly appreciate the financial support from the National Natural Science Foundation of China (Nos.21425103, 21673280, 31470931 and 91527302), the Key Research Program of the Chinese Academy of Sciences (No.KGZD-EW-T02-3) and Wuhan Huanghe Talents Program of Science and Technology.

\section{Competing Interests}

The authors have declared that no competing interest exists.

\section{References}

1. Li F, Wang $\mathrm{O}$. Fabrication of nanoarchitectures templated by virus-based nanoparticles: strategies and applications. Small. 2014; 10: 230-245.

2. Steinmetz NF. Viral nanoparticles as platforms for next-generation therapeutics and imaging devices. Nanomed Nanotechnol Biol Med. 2010; 6: 634-641.

3. Wen AM, Steinmetz NF. Design of virus-based nanomaterials for medicine, biotechnology, and energy. Chem Soc Rev. 2016; 45: 4074-4126.

4. Kerfeld CA, Melnicki MR. Assembly, function and evolution of cyanobacterial carboxysomes. Curr OpinPlant Biol. 2016; 31: 66-75.

5. Kerfeld CA, Heinhorst S, Cannon GC. Bacterial microcompartments. Annu Rev Microbiol. 2010; 64: 391-408.

6. Theil EC. Ferritin: structure, gene regulation, and cellular function in animals, plants, and microorganisms. Annu Rev Biochem. 1987; 56: 289-315.
7. Rivera M. Bacterioferritin: structure, dynamics, and protein-protein interactions at play in iron storage and mobilization. Accounts Chem Res. 2017; 50: 331-340.

8. Bronstein LM. Virus-based nanoparticles with inorganic cargo: What does the future hold? Small. 2011; 7: 1609-1618.

9. Schwarz B, Douglas T. Development of virus-like particles for diagnostic and prophylactic biomedical applications. WIREs Nanomed Nanobiotechnol. 2015; 7: $722-735$.

10. Douglas T, Young M. Host-guest encapsulation of materials by assembled virus protein cages. Nature. 1998; 393: 152-155.

11. Klem MT, Young M, Douglas T. Biomimetic synthesis of $\beta-\mathrm{TiO}_{2}$ inside a viral capsid. J Mater Chem. 2008; 18: 3821-3823.

12. Douglas T, Strable E, Willits D, et al. Protein engineering of a viral cage for constrained nanomaterials synthesis. Adv Mater. 2002; 14: 415-418.

13. Aljabali AA, Sainsbury F, Lomonossoff GP, et al. Cowpea mosaic virus unmodified empty viruslike particles loaded with metal and metal oxide. Small. 2010; 6: 818-821.

14. Jaafar M, Aljabali AAA, Berlanga I, et al. Structural insights into magnetic clusters grown inside virus capsids. ACS Appl Mater Interfaces. 2014; 6: 20936-20942.

15. Dujardin E, Peet C, Stubbs G, et al. Organization of metallic nanoparticles using tobacco mosaic virus templates. Nano Lett. 2003; 3: 413-417.

16. Kobayashi $\mathrm{M}$, Seki $\mathrm{M}$, Tabata $\mathrm{H}$, et al. Fabrication of aligned magnetic nanoparticles using tobamoviruses. Nano Lett. 2010; 10: 773-776.

17. Reichhardt C, Uchida M, O'Neil A, et al. Templated assembly of organic-inorganic materials using the core shell structure of the P22 bacteriophage. Chem Commun. 2011; 47: 6326-6328.

18. Bedwell GJ, Zhou Z, Uchida M, et al. Selective biotemplated synthesis of $\mathrm{TiO}_{2}$ inside a protein cage. Biomacromolecules. 2014; 16: 214-218.

19. Zhou Z, Bedwell GJ, Li R, et al. Formation mechanism of chalcogenide nanocrystals confined inside genetically engineered virus-like particles. Sci Rep. 2013; 4: 3832-3832.

20. Zhou Z, Bedwell GJ, Li R, et al. P22 virus-like particles constructed Au/CdS plasmonic photocatalytic nanostructures for enhanced photoactivity. Chem Commun. 2015; 51: 1062-1065. 
21. Zhou K, Li F, Dai G, et al. Disulfide bond: dramatically enhanced assembly capability and structural stability of tobacco mosaic virus nanorods. Biomacromolecules. 2013; 14: 2593-2600.

22. Zhou K, Zhang JT, Wang QB. Site-selective nucleation and controlled growth of gold nanostructures in tobacco mosaic virus nanotubulars. Small. 2015; 11: 2505-2509.

23. Tsukamoto R, Muraoka M, Seki $\mathrm{M}$, et al. Synthesis of $\mathrm{CoPt}$ and $\mathrm{FePt}_{3}$ nanowires using the central channel of tobacco mosaic virus as a biotemplate. Chem Mater. 2007; 19: 2389-2391.

24. Knez M, Bittner AM, Boes F, et al. Biotemplate synthesis of 3-nm nickel and cobalt nanowires. Nano Lett. 2003; 3: 1079-1082.

25. Kadri A, Maiss E, Amsharov N, et al. Engineered tobacco mosaic virus mutants with distinct physical characteristics in planta and enhanced metallization properties. Virus Res. 2011; 157: 35-46.

26. Balci $S, H a h n ~ K$, Kopold $P$, et al. Electroless synthesis of $3 \mathrm{~nm}$ wide alloy nanowires inside tobacco mosaic virus. Nanotechnology. 2012; 23: 045603.

27. Balci S, Bittner AM, Hahn $\mathrm{K}$, et al. Copper nanowires within the central channel of tobacco mosaic virus particles. Electrochimica Acta. 2006; 51: 6251-6257.

28. Zhang W, Zhang Z-P, Zhang X-E, et al. Reaction inside a viral protein nanocage: mineralization on a nanoparticle seed after encapsulation via self-assembly. Nano Res. 2017. doi: 10.1007/s12274-017-1541-3.

29. Jutz G, van Rijn P, Santos Miranda B, et al. Ferritin: a versatile building block for bionanotechnology. Chem Rev. 2015; 115: 1653-1701.

30. Uchida M, Klem MT, Allen M, et al. Biological containers: protein cages as multifunctional nanoplatforms. Adv Mater. 2007; 19: 1025-1042.

31. Chen C, Daniel MC, Quinkert ZT, et al. Nanoparticle-templated assembly of viral protein cages. Nano Lett. 2006; 6: 611-615.

32. Dixit SK, Goicochea NL, Daniel MC, et al. Quantum dot encapsulation in viral capsids. Nano Lett. 2006; 6: 1993-1999.

33. Daniel MC, Tsvetkova IB, Quinkert ZT, et al. Role of surface charge density in nanoparticle-templated assembly of bromovirus protein cages. ACS Nano. 2010; 4: 3853-3860.

34. Kusters $\mathrm{R}$, Lin $\mathrm{H}-\mathrm{K}$, Zandi $\mathrm{R}$, et al. Role of charge regulation and size polydispersity in nanoparticle encapsulation by viral coat proteins. J Phys Chem B. 2015; 119: 1869-1880

35. Li F, Li K, Cui ZQ, et al. Viral coat proteins as flexible nano-building-blocks for nanoparticle encapsulation. Small. 2010; 6: 2301-2308.

36. Liddington RC, Yan Y, Moulai J, et al. Structure of simian virus-40 at 3.8-A resolution. Nature. 1991; 354: 278-284.

37. Loo L, Guenther RH, Basnayake VR, et al. Controlled encapsidation of gold nanoparticles by a viral protein shell. J Am Chem Soc. 2006; 128: 4502-4503.

38. Loo L, Guenther RH, Lommel SA, et al. Encapsidation of nanoparticles by red clover necrotic mosaic virus. J Am Chem Soc. 2007; 129: 11111-11117.

39. Zhang Y, Ke X, Zheng Z, et al. Encapsulating quantum dots into enveloped virus in living cells for tracking virus infection. ACS Nano. 2013; 7: 3896-3904.

40. Li Q, Li W, Yin W, et al. Single particle tracking of human immunodeficiency virus type 1 productive entry into human primary macrophages. ACS Nano. 2017; 11: 3890-3903.

41. Shen $\mathrm{L}$, Zhou J, Wang Y, et al. Efficient encapsulation of $\mathrm{Fe}_{3} \mathrm{O}_{4}$ nanoparticles into genetically engineered hepatitis B core virus-like particles through a specific interaction for potential bioapplications. Small. 2015; 11: 1190-1196.

42. Sun J, DuFort C, Daniel M-C, et al. Core-controlled polymorphism in virus-like particles. Proc Natl Acad Sci. 2007; 104: 1354-1359.

43. Huang X, Bronstein LM, Retrum J, et al. Self-assembled virus-like particles with magnetic cores. Nano Lett. 2007; 7: 2407-2416.

44. He L, Porterfield Z, van der Schoot $P$, et al. Hepatitis virus capsid polymorph stability depends on encapsulated cargo size. ACS Nano. 2013; 7: 8447-8454.

45. Blanco E, Shen H, Ferrari M. Principles of nanoparticle design for overcoming biological barriers to drug delivery. Nat Biotechnol. 2015; 33: 941-951.

46. Tsvetkova I, Chen C, Rana S, et al. Pathway switching in templated virus-like particle assembly. Soft Matter. 2012; 8: 4571-4577.

47. Ntziachristos V. Fluorescence molecular imaging. Annu Rev Biomed Eng. 2006; 8: 1-33.

48. Pinaud F, Michalet X, Bentolila LA, et al. Advances in fluorescence imaging with quantum dot bio-probes. Biomaterials. 2006; 27: 1679-1687.

49. Li F, Zhang Z-P, Peng J, et al. Imaging viral behavior in mammalian cells with self-assembled capsid-quantum-dot hybrid particles. Small. 2009; 5: 718-726.

50. Li C, Li F, Zhang Y, et al. Real-time monitoring surface chemistry-dependent in vivo behaviors of protein nanocages via encapsulating an NIR-II $\mathrm{Ag}_{2} \mathrm{~S}$ quantum dot. ACS Nano. 2015; 9: 12255-12263.

51. Gustav JS, Willem JMM, Geralda AFvT, et al. MRI contrast agents: current status and future perspectives. Anticancer Agents Med Chem. 2007; 7: 291-305.

52. Liepold L, Anderson S, Willits D, et al. Viral capsids as MRI contrast agents. Magn Reson Med. 2007; 58: 871-879.

53. Allen M, Bulte JWM, Liepold L, et al. Paramagnetic viral nanoparticles as potential high-relaxivity magnetic resonance contrast agents. Magn Reson Med. 2005; 54: 807-812

54. Michael $A B$, Xin $Y$, Nicole FS. Engineering Gd-loaded nanoparticles to enhance MRI sensitivity via T 1 shortening. Nanotechnology. 2013; 24: 462001.

55. Shukla S, Steinmetz NF. Virus-based nanomaterials as positron emission tomography and magnetic resonance contrast agents: from technology development to translational medicine. WIREs Nanomed Nanobiotechnol. 2015; 7: 708-721.
56. Huang $\mathrm{X}$, Stein BD, Cheng $\mathrm{H}$, et al. Magnetic virus-like nanoparticles in $N$. benthamiana plants: a new paradigm for environmental and agronomic biotechnological research. ACS Nano. 2011; 5: 4037-4045.

57. Malyutin AG, Easterday R, Lozovyy Y, et al. Viruslike nanoparticles with maghemite cores allow for enhanced MRI contrast agents. Chem Mater. 2015; 27: 327-335.

58. Kaneda Y, Nakajima T, Nishikawa T, et al. Hemagglutinating virus of Japan (HVJ) envelope vector as a versatile gene delivery system. Mol Ther. 2002; 6: 219-226

59. Sun $X$, Li $W$, Zhang $X$, et al. In vivo targeting and imaging of atherosclerosis using multifunctional virus-like particles of simian virus 40. Nano Lett. 2016; 16: 6164-6171.

60. Dragnea B, Chen C, Kwak E-S, et al. Gold nanoparticles as spectroscopic enhancers for in vitro studies on single viruses. J Am Chem Soc. 2003; 125: 6374-6375.

61. Li F, Gao D, Zhai $\mathrm{X}$, et al. Tunable, discrete, three-dimensional hybrid nanoarchitectures. Angew Chem Int Ed. 2011; 50: 4202-4205.

62. Capehart SL, Coyle MP, Glasgow JE, et al. Controlled integration of gold nanoparticles and organic fluorophores using synthetically modified MS2 viral capsids. J Am Chem Soc. 2013; 135: 3011-3016.

63. Aniagyei SE, Kennedy CJ, Stein B, et al. Synergistic effects of mutations and nanoparticle templating in the self-assembly of cowpea chlorotic mottle virus capsids. Nano Lett. 2009; 9: 393-398.

64. Liu AJ, Traulsen $\mathrm{CHH}$, Cornelissen JJLM. Nitroarene reduction by a virus protein cage based nanoreactor. ACS Catal. 2016; 6: 3084-3091.

65. Liu AJ, Verwegen M, de Ruiter MV, et al. Protein cages as containers for gold nanoparticles. J Phys Chem B. 2016; 120: 6352-6357.

66. Chen C, Kwak ES, Stein B, et al. Packaging of gold particles in viral capsids. J Nanosci Nanotechnol. 2005; 5: 2029-2033.

67. Ashley CE, Carnes EC, Phillips GK, et al. Cell-specific delivery of diverse cargos by bacteriophage MS2 virus-like particles. ACS Nano. 2011; 5: 5729-5745

68. Enomoto T, Kawano M, Fukuda H, et al. Viral protein-coating of magnetic nanoparticles using simian virus 40 VP1. J Biotechnol. 2013; 167: 8-15.

69. Li F, Chen H, Zhang Y, et al. Three-dimensional gold nanoparticle clusters with tunable cores templated by a viral protein scaffold. Small. 2012; 8: 3832-3838.

70. Gao D, Lin XP, Zhang ZP, et al. Intracellular cargo delivery by virus capsid protein-based vehicles: From nano to micro. Nanomedicine. 2016; 12: 365-376.

71. Wang T, Zhang Z, Gao D, et al. Encapsulation of gold nanoparticles by simian virus 40 capsids. Nanoscale. 2011; 3: 4275-4282.

72. Malyutin AG, Cheng H, Sanchez-Felix OR, et al. Coat protein-dependent behavior of poly(ethylene glycol) tails in iron oxide core virus-like nanoparticles. ACS Appl Mater Interfaces. 2015; 7: 12089-12098.

73. Goicochea NL, De M, Rotello VM, et al. Core-like particles of an enveloped animal virus can self-assemble efficiently on artificial templates. Nano Lett. 2007; 7: 2281-2290 